\title{
Clinical Study \\ Single-Port Laparoscopic Cholecystectomy Using the Innovative E. K. Glove Port: Our Experience
}

\author{
Elbert Khiangte, ${ }^{1}$ Iheule Newme, ${ }^{1}$ Karabi Patowary, ${ }^{1}$ and Hitesh Kalita ${ }^{2}$ \\ ${ }^{1}$ International Hospital, Guwahati, Assam 781005, India \\ ${ }^{2}$ Diphu Civil Hospital, Diphu, Assam 782460, India
}

Correspondence should be addressed to Elbert Khiangte, ekhiangte@yahoo.in

Received 24 March 2012; Accepted 22 April 2012

Academic Editors: F. Agresta and A. Umezawa

Copyright ( $) 2012$ Elbert Khiangte et al. This is an open access article distributed under the Creative Commons Attribution License, which permits unrestricted use, distribution, and reproduction in any medium, provided the original work is properly cited.

\begin{abstract}
The technique of laparoscopic cholecystectomy continues to evolve with a trend towards decreasing use of working ports. One of the emerging concepts of 21st century is single-port surgery. It has further minimized the minimally invasive surgery. However, the main drawbacks of this technique are the lack of "triangulation" to which the laparoscopic surgeons have grown accustomed to, the clustering of instruments, and the costly multichannel ports, which are very costly and, in fact, are not affordable by the majority of the population in a developing country like India. From September 2009 to December 2011, 210 patients identified as having biliary colic, chronic cholecystitis, and previous biliary pancreatitis or obstructive jaundice due to stones (managed by ERCP) underwent single-port laparoscopic cholecystectomy using the E. K. glove port. The operating time was reasonable and can be lessened with experience. Excellent exposure of the critical view was obtained in all cases. This technique is safe, feasible, reproducible, cheap, and easy to learn. It may be an alternative to the currently available single-port access system, especially in a developing country like India. If required, placement of the remaining two to three ports for a more conventional laparoscopic cholecystectomy can be done.
\end{abstract}

\section{Introduction}

In an effort to reduce morbidity and improve the cosmesis of laparoscopic surgery, surgeons have tried to reduce the size and number of ports. Single-port surgery has recently emerged, where the surgery is done through a single-port, typically the patient's navel. This improves the cosmesis, lessens post-operative pain, and ensures virtually a "scarless" surgery.

Single-port laparoscopic cholecystectomy (SPLC) is perhaps the most common single-port surgery procedure used to treat patients with gall stone diseases. SPLC can be performed using (a) one of the many commercially available multichannel single-port devices: R-port (Advanced Surgical Concepts, Dublin, Ireland), XCONE (Karl Storz, Tuttlingen, Germany), SILS port (Covidien), and SPIDER (TransEnterix, Durham, NC, USA); (b) passing three $5 \mathrm{~mm}$ trocars side by side through the fascia via a single umbilical incision; (c) using an extra-small wound retractor (ALEXIS wound retractor XS, Applied Medical) and a surgical glove as the "single port" through the umbilical incision.

In this paper, we report our experience with 210 patients who underwent SPLC and a detailed description of the technique with special reference to the E. K. glove port [1], our evaluation of retrospective examination of prospectively collected data of patients operated by a single surgeon, main author (E. Khiangte). The aim of this paper is to encourage laparoscopic surgeons, especially in developing countries, to adopt our technique of SPLC using the cost-effective E. K. glove port.

\section{Materials and Methods}

2.1. Patients. From September 2009 to December 2011, 210 patients (90 males and 120 females) identified as having biliary colic, chronic cholecystitis, and previous biliary pancreatitis or obstructive jaundice due to stones (managed 
by ERCP) were prepared for an elective cholecystectomy. The diagnosis was mainly based on abdominal ultrasonography or computed tomography. Most of the patients had subjective symptoms such as right upper quadrant abdominal pain, dyspepsia, flatulence or abdominal discomfort. Cases of acute cholecystitis requiring emergency cholecystectomy, gallbladder polyps, cholelithiasis with choledocholithiasis requiring $\mathrm{CBD}$ exploration, suspicion of $\mathrm{GB}$ malignancies, and extensive previous abdominal surgeries; patients who were of high risk for general anesthesia and obese patients were excluded from this study. Patients who required extraumbilical incisions due to technical difficulties were termed as 2-port or 3-port surgery and were not included in this study.

All patients were informed in great detail during the informed consent process about the laparoscopic technique of having a single incision in the abdomen with a possibility of several more incisions or even conversion to an open technique based on the operative findings and feasibility. No patients refused to undergo such a technique. No institutional review board approval was sought because the technique change, in our opinion, was akin to simple port repositioning, which did not constitute an experimental protocol [2].

2.2. The E. K. Port. Materials required to make the E. K. glove port are (Figure 1)

(1) A flexible rubber inner ring (diameter 5-6 cm),

(2) A plastic rigid outer ring (diameter $11-12 \mathrm{~cm}$ ),

(3) A pair of surgical gloves,

(4) Standard laparoscopic trocars or low-profile laparoscopic trocars.

2.3. Preparation of the E. K. Glove Port. The finger of one of the gloves was cut into several thin rings, to be used as rubber bands. The fingers of the other glove were truncated with scissors and the trocars were fitted into it and fixed with the rubber bands made earlier (Figure 2). We usually use two $5 \mathrm{~mm}$ and two $10 \mathrm{~mm}$ ports: $5 \mathrm{~mm}$ ports for hand instruments and $10 \mathrm{~mm}$ ports for the laparoscope and clip applicator for larger cystic duct clipping.

The open end of the glove was passed through the flexible inner ring (Figure 3) and turned over the ring so that the flexible ring was between the two layers of the glove (Figure 4).

2.4. Operative Technique. All patients underwent general anesthesia and, after intubation, were positioned supine on the operating table. Both the upper extremities were abducted and placed on arm boards at an angle of less than $90^{\circ}$ to the body. A preoperative dose of a cephalosporin was administered after negative skin test. The abdomen was prepared with savlon, betadine, and spirit and draped with sterile linen.

The surgeon stood on the left side of the patient with the assistant on the left side of the surgeon. The scrub

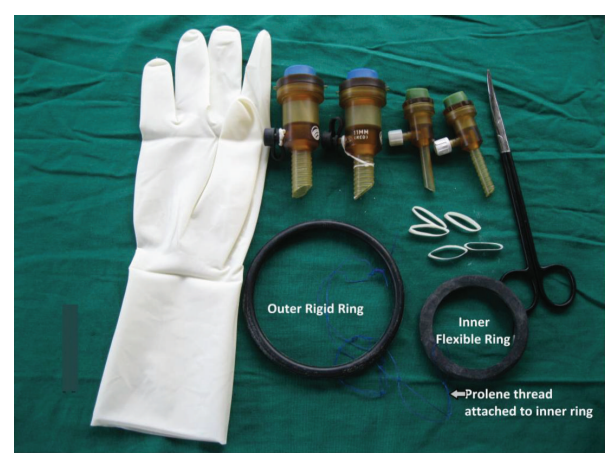

FIgURE 1: Materials required for making the E. K. Glove port.

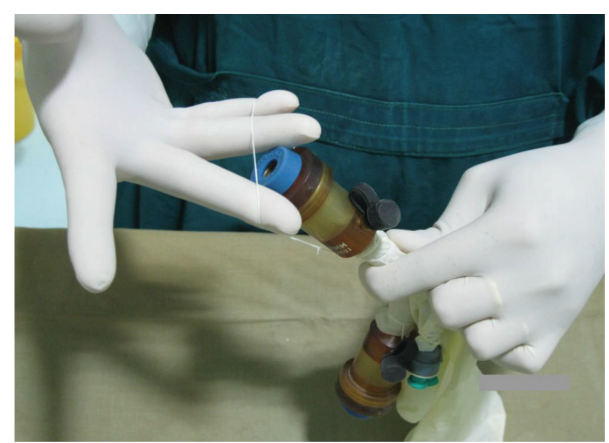

Figure 2: Trocar fixed to the glove with rubber bands.

nurse stood on the right side of the patient (Figure 5). The monitor trolley was placed above the patient's right arm. We routinely used a standard $10 \mathrm{~mm}, 30^{\circ}$ angled rigid laparoscope. This decision was based purely on the nonavailability of a special laparoscope in our operating room. We routinely use roticulator or curved graspers for the left hand, standard rigid $5 \mathrm{~mm}$ laparoscopic instruments for the right hand, and standard reusable $10 \mathrm{~mm}$ and/or $5 \mathrm{~mm}$ clip applicators (for LT-400, LT-300, and LT-200 clips) for all procedures.

The umbilicus was everted by pulling the deepest point of the umbilical scar out of its normal-indented position. A 1.5$2.0 \mathrm{~cm}$ completely intraumbilical, vertical curvilinear skin incision without the extension of this incision beyond the outer limits of the umbilical folds was performed (Figure 6). The incision was deepened, and a $2.0-2.5 \mathrm{~cm}$ rectus fasciotomy was made to enter the peritoneal cavity. The inner flexible ring, fitted with the glove, was then introduced into the abdomen assisted by a retractor and fingers (Figure 7). The outer rigid ring was placed over the glove, and the open end of the glove was then wrapped around the outer rigid ring (Figure 8). $\mathrm{CO}_{2}$ pneumoperitoneum was induced and maintained at 12-14 $\mathrm{mm}$ of $H_{\mathrm{g}}$. The patients were then put in reverse Trendelenburg position and tilted slightly left laterally for the remainder of the procedure.

We routinely used an 18-gauze lumbar puncture (LP) needle through the right hypochondrium just below the ribs which served to aspirate the liquid contents of the gallbladder in selected cases. The needle was then curved 


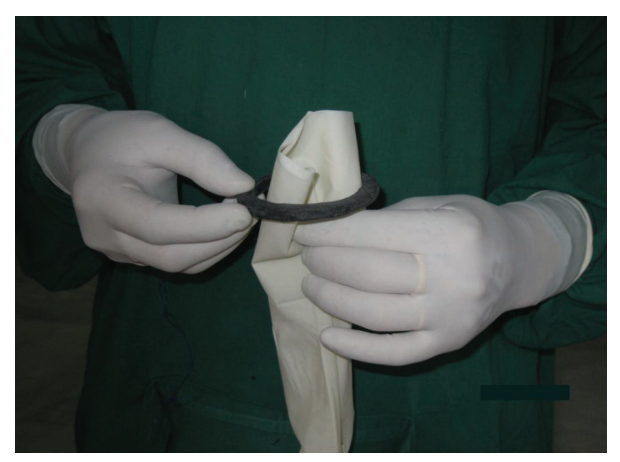

FIGURE 3: Open end of the glove passed through the inner flexible ring.

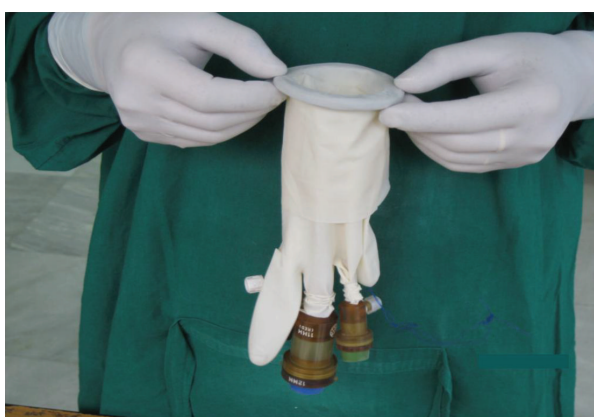

Figure 4: Flexible ring between the two layers of the glove.

with a needle holder to form a hook. It was then used to hook the fundus of the gallbladder and retract it. If one is gentle enough to hook only the serosa and wall of the gallbladder, inadvertent bile leakage can be avoided. The LP needle was then pulled from outside and fixed with a hemostat to get a fairly good retraction (Figure 9). Occasionally, when the liver margin and the gallbladder were found to be high above the level of costal margin, a no. 1-0 nylon suture with a straight needle was used to retract the fundus of the gallbladder. A tuft or bun was made with the tail end of the suture. The needle was passed through the fundus of the gallbladder, care being taken to pass the needle through the serosa without puncturing the lumen. It was next passed through the peritoneum below the diaphragm. The needle was then brought out through the right subcostal region. When the thread was pulled from outside and fixed with a hemostat, we get good retraction of the gallbladder imitating retraction in accordance to conventional technique for safe cholecystectomy (Figure 10). The addition of a needle or suture was not considered as a deviation from the single port as it required only a puncture and not an incision [3].

With the left-hand dissector, the infundibulum of the gallbladder was grasped and retracted superolaterally to expose the Calot's triangle. With the help of a monopolar diathermy hook on the right hand, the posterior peritoneum was divided to free the Hartmann's pouch. This was followed by further dissection of the anterior and posterior peritoneal leaves overlying the Calot's triangle. Using a hook and/or Maryland dissector with the right hand, the cystic duct and

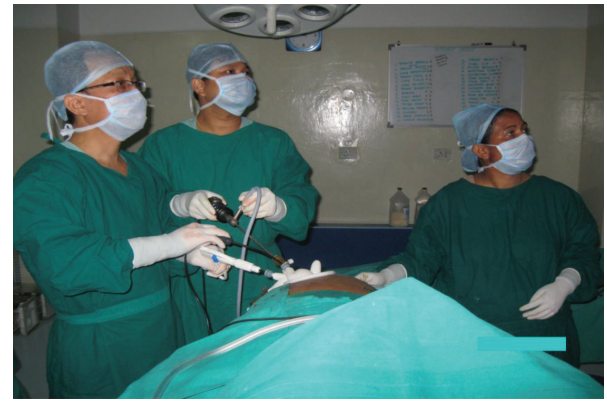

Figure 5: Position of the surgeon, assistant surgeon, and the scrub nurse.

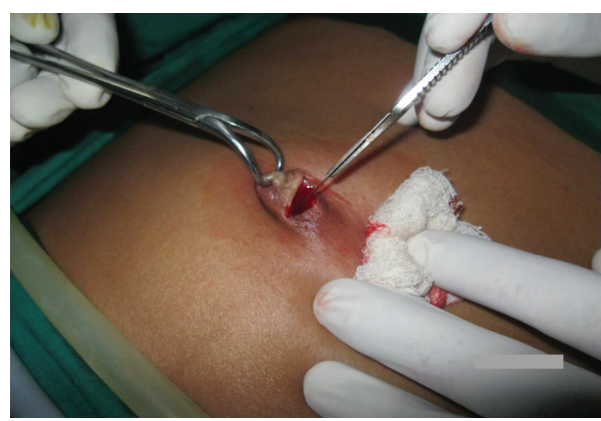

Figure 6: Incision in the umbilicus.

artery were clearly skeletonized till the triangulation of cystic duct, common bile duct, and the liver's edge was achieved. We prefer a large "window" in the Calot's triangle so as to safely observe the tip of the clip applicators while clipping the structures. The proximal cystic artery was double clipped using a $5 \mathrm{~mm}$ reusable clip applicator, and divided with a monopolar hook. The proximal cystic duct was doubly clipped using a reusable $10 \mathrm{~mm}$ clip applicator, and a third clip was placed as high as possible toward the gallbladder, and the cystic duct was divided with a pair of scissors. We do not change to $5 \mathrm{~mm}$ telescope while using $10 \mathrm{~mm}$ clip applicator as the E. K. port [1] can accommodate both the $10 \mathrm{~mm}$ instruments with ease. For two of our patients, with wide cystic duct, intracorporeal suturing was done with a no. 1 polyglactin suture to tie the cystic duct. Two knots were applied proximally and one distally toward the gallbladder, and the cystic duct was divided with scissors (Figure 11).

Next, the gallbladder was grasped with the left-hand instrument and retracted in various directions so that it could be dissected from the liver bed by hook electrocautery in an infundibulum-to-fundal direction. Prior to the final detachment of the gallbladder, meticulous haemostasis of the liver bed was confirmed. After the complete dissection of the gallbladder, it was kept hanging on the anterior abdominal wall with the help of the LP needle hook or the nylon thread while suction and irrigation was done, if required, to achieve adequate peritoneal toilette. No pre-operative cholangiogram was performed in this series.

We used a sterile, inexpensive plastic pouch with a purse string suture to retrieve the specimen. The pouch was rolled up, introduced into the abdomen with the help of a grasper 


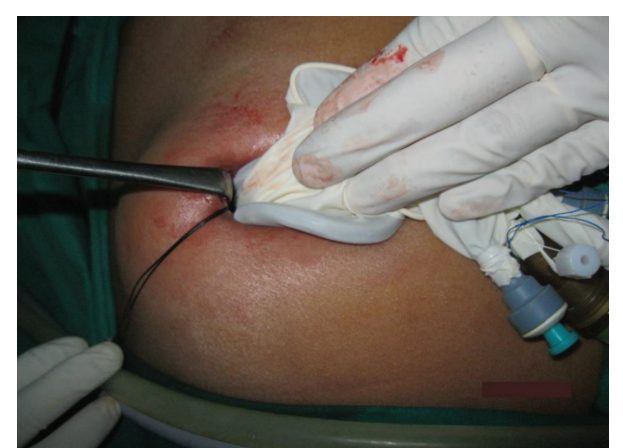

FIGURE 7: Inner flexible ring along with the glove being introduced into the abdomen.

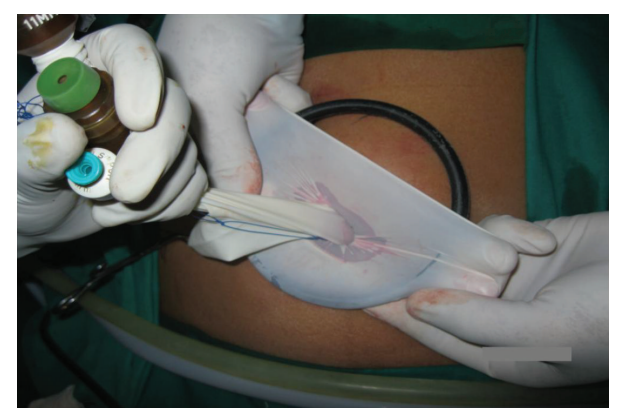

FIGURE 8: Open end of the glove wrapped around the outer rigid ring.

through the $10 \mathrm{~mm}$ port, and opened on the superior surface of the liver. After the introduction of the specimen, the mouth of the bag was closed by pulling the purse string. The end of the purse string suture was then held by a grasper and pulled into the port, and pneumoperitoneum was deflated. An incision was made in the glove which facilitated extraction of the bag with the specimen, and the specimen was sent for histopathology.

Removal of the E. K. port [1] was by simply pulling the prolene thread attached to the inner ring. The fascial defect was closed using 1-0 Polydioxanone (Ethicon), and the skin was approximated using 3-0 polyglycolic Rapide suture (Ethicon) (Figure 12). Packing a small gauze ball beneath a dressing was sufficient to restore the natural scar of the umbilicus. We routinely applied a compression bandage around the umbilicus which helped to minimize seroma formation. The patients were extubated in the operating room and brought to the postanesthesia care unit.

2.5. The Learning Curve. Initially, we trained ourselves with "two ports" placing the E. K. Port [1] in the umbilicus and a $5 \mathrm{~mm}$ port in the epigastrium. The telescope, fundal retractor, and the left-hand grasper were passed through the E. K. port [1], and a $5 \mathrm{~mm}$ epigastrium port was used to do the dissections. All $10 \mathrm{~mm}$ instruments were passed through the E. K. port [1]. After successfully completing 20 "two-port" surgeries using this port, we performed the first single-port surgery in September 2009. This method, we believe, boosts our confidence and a subjective sense

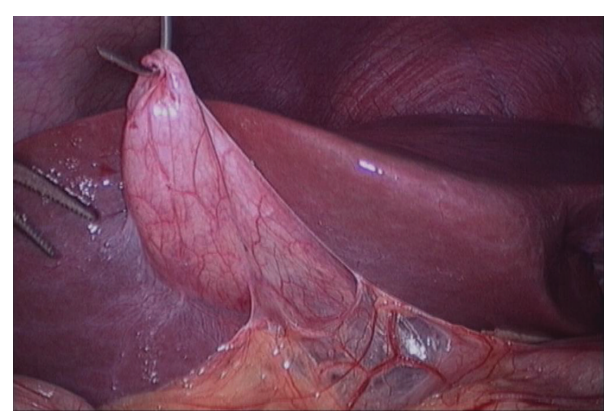

FIGURE 9: Lumber puncture needle hook used as a fundal retractor.

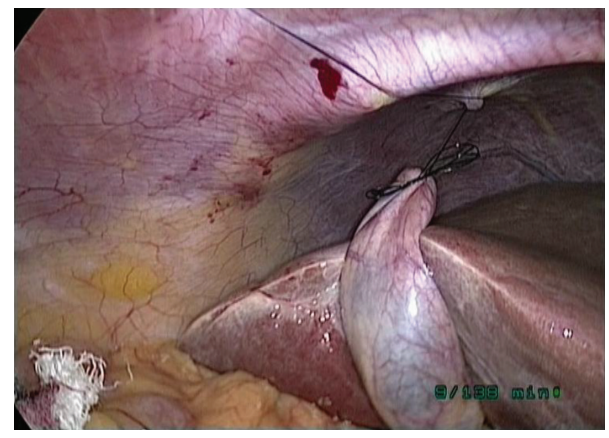

FIGURE 10: Nylon thread used as a fundal retractor.

of improved feasibility and security and also shortens the learning curve. We have observed that experienced laparoscopic surgeons may not need to undergo a steep learning curve, especially when the basic concepts of this emergent technique are understood: its inherent challenges and implementation of potential solutions. We successfully performed 210 single-port cholecystectomies using the E. K. port. The mean operating time was $60.8 \mathrm{~min}$ (range, 30-125 min) which is comparable with other published series $[2,4,5]$. Although this procedure took longer than conventional laparoscopic cholecystectomy, the operation time was significantly reduced as we gained experience and confidence. A gradual learning curve of 20 to 30 surgeries may be suggested for a surgeon to safely adopt the procedure in clinical practice $[4,5]$.

\section{Results}

Single-port cholecystectomy was successfully completed in 210 patients using this technique from September 2009 to December 2011. A total of 120 female (57.14\%) and 90 male $(42.86 \%)$ patients between the age group of $12-65$ yrs underwent this procedure.

All the surgeries were completed without any intraoperative or postoperative complications. Early in our series, two patients required an additional $5 \mathrm{~mm}$ port in the epigastrium due to poor visualization of Calot's triangle, and one patient required another $5 \mathrm{~mm}$ port in the right hypochondrium due to bleeding. These procedures were termed as 2-port and 3port surgery and were not included in the present study. The low conversion rate in this series may be attributed to our 


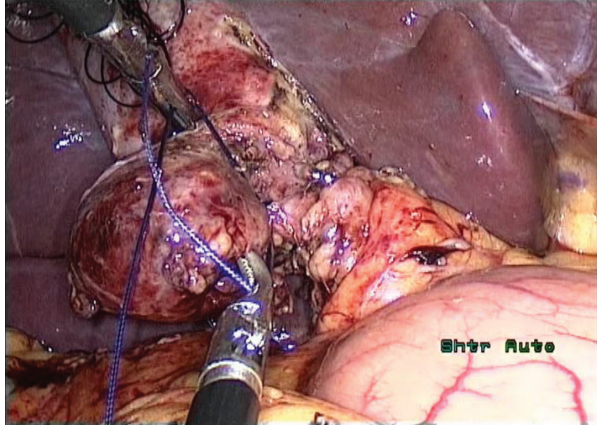

FIGURE 11: Polyglactin suture is used to tie the wide cystic duct.

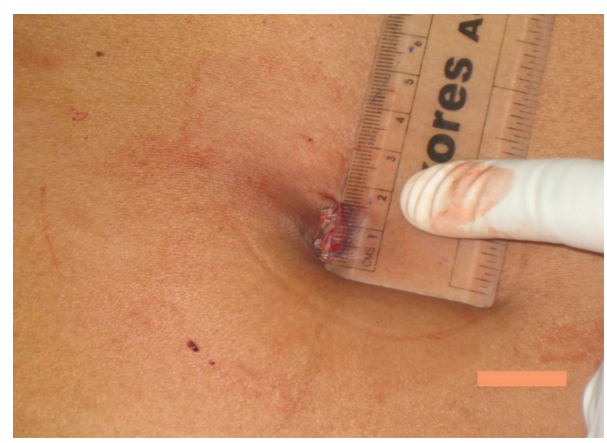

Figure 12: The skin incision about $1.5 \mathrm{~cm}$ was repaired with polyglycolic Rapide suture (Ethicon).

one skin incision in the umbilicus and three transabdominal gallbladder stay sutures [10]. Piskun and Rajpal used the multiple puncture method to perform laparoscopic cholecystectomy [11]. Raman et al. used this method to perform nephrectomy [12]. However, the use of three different fascial incisions could become a problem with a gas leak [4], skin maceration, and fascial tear and further complicate wound healing [13]. There is also likelihood of higher incidence of port-site hernias due to the use of multiple closely placed fascial incisions through a narrow area. A "Swiss-cheese" configuration of $5 \mathrm{~mm}$ fascial defects and pressure necrosis of the tissues due to placement of tight-fitting access devices are factors to be considered [14].

The commercially available single-port access system like the R-port, X-Cone, and SILS, and so forth are designed specifically for single-port surgery. Various operations like cholecystectomy, sigmoidectomy, and nephrectomy have been reported using these single-port access systems [15-17].

Homemade transumbilical port using Alexis wound retractor, glove, and conventional laparoscopic trocars has been in use to perform various operations like cholecystectomy, nephrectomy, appendectomy, transumbilical preperitoneal (TAPP) inguinal hernia repair, varicocelectomy, and hemicolectomy $[13,18-20]$.

The improvised E. K. glove port [1] is very cheap in comparison to the commercially available single-port access system and is more cost-effective than the homemade transumbilical port where an Alexis wound retractor or fixation to the fascial edge is required $[5,13,18,19,21,22]$. A variety of instruments ( 3 to $12 \mathrm{~mm}$ ) can be used to facilitate the procedure. The number of trocars to be used can be planned preoperatively or replace the smaller trocars to larger one or vice versa as per demand of the surgery. In addition, the glove acts as a wound protector and avoids port site contamination while retrieving infected or malignant specimens. It can also prevent subcutaneous emphysema as well as port-site bleeding due to the tamponade effect of the inner and outer rings. No gas leakage was noted during the procedure. However, one should be cautious while introducing sharp instruments for fear of tearing the glove. The E. K. port is simple and is made of easily available materials, making it unnecessary to purchase any expensive new devices. 
In SPLC, all instruments are passed through a single port in the umbilicus, and they are parallel to each other so the concept of triangulation, thought to be the basis of laparoscopic surgery, is hard to achieve. Instrument handle clashing, reduced operative work space, inadequate retraction, increased operating time, and compromised view are other problems. The counterintuitive movements due to frequent crossing of the instrument shafts at the point of entry into the abdominal cavity are another major hurdle in single-port surgery.

We employed a curved or a roticulator grasper for the left hand to achieve some degree of triangulation, but in order to deliver sufficient torque or power of the surgeon to push and pull the tissue, we used conventional straight laparoscopic instruments for the right hand. It was our observation that the E. K. port promises a more streamlined process, with the benefit of a bimanual performance by the surgeon without crossing the instruments. SPLC can be performed with a combination of conventional straight instruments, a roticulator, or curved hand instruments.

The average age of the patients in our study was 33.6 years (range, 12-65 years), and the mean operating time was $60.8 \mathrm{~min}$ (range, 30-125 min) which is almost similar to other series $[2,4,5]$. We observed that the perception of pain was comparable to the conventional laparoscopic surgery on the day of operation (day-0) but was comparatively lesser on the following postoperative days.

The eagerness of the patient to have a virtually scarless surgery is very high. The bemused postoperative look on the patient's face to know that the "existing scar," the umbilicus, was used for the surgery belittles all other pleasure for the surgeon. It is worth the patience, time, and energy spent to see a satisfied patient.

Conventional laparoscopic cholecystectomy being time tested, the connoisseurs of SPLC do not feel the necessity to try and establish supremacy, but keeping in view the changing trends in the field of surgery, comparative studies can provide a newer variant and technique of laparoscopic cholecystectomy with all its advantages.

\section{Conclusion}

Laparoscopic cholecystectomy is one of the commonest operations performed worldwide. SPLC appears to be cosmetically superior to standard laparoscopic cholecystectomy. We utilize the body's natural scar, the umbilicus to create a scar. We do not make a new scar. SPLC was found to be technically feasible and safe in patients with noncomplicated gall stone diseases. The SPLC technique with the innovative E. K. glove port is simple, reusable, costeffective, safe, reproducible, and a reliable gadget for singleport cholecystectomy. It may be an alternative to the costly, commercially available single-port system, especially in a developing country like India. The total cost reduction cannot be ignored, and this, along with its safety and simplicity, would be one more essential reason for its use. Wider use of this technique will definitely help the surgeons to take single-port access to the masses. If required, placement of the remaining two to three ports for a more conventional laparoscopic cholecystectomy can be done. The operating time was reasonable and can be lessened with experience. The SPLC procedure using the E. K. port is becoming the standard of care for most of the authors' elective patients with gallbladder diseases. Clinical trials are warranted before the SPLC technique is adopted universally.

\section{Conflict of Interests}

E. Khiangte, I. Newme, K. Patowary, and H. Kalita have no conflict of interests or financial ties to disclose. They do not have any financial relation with the commercial identity mentioned in this paper.

\section{References}

[1] E. Khiangte, I. Newme, P. Phukan, and S. Medhi, "Improvised transumbilical glove port: a cost effective method for single port laparoscopic surgery," Indian Journal of Surgery, vol. 73, no. 2, pp. 142-145, 2011.

[2] A. C. Petrotos and B. M. Molinelli, "Single-incision multiport laparoendoscopic (SIMPLE) surgery: early evaluation of SIMPLE cholecystectomy in a community setting," Surgical Endoscopy, vol. 23, no. 11, pp. 2631-2634, 2009.

[3] A. Meining, D. Wilhelm, M. Burian et al., "Development, standardization, and evaluation of NOTES cholecystectomy using a transsigmoid approach in the porcine model: an acute feasibility study," Endoscopy, vol. 39, no. 10, pp. 860-864, 2007.

[4] H. Rivas, E. Varela, and D. Scott, "Single-incision laparoscopic cholecystectomy: initial evaluation of a large series of patients," Surgical Endoscopy, vol. 24, no. 6, pp. 1403-1412, 2010.

[5] E. J. Koo, S. H. Youn, Y. H. Baek et al., "Review of 100 cases of single port laparoscopic cholecystectomy," Journal of the Korean Surgical Society, vol. 82, no. 3, pp. 179-184, 2012.

[6] T. Kagaya, "Laparoscopic cholecystectomy via two ports, using the "Twin-Port" system," Journal of Hepato-Biliary-Pancreatic Surgery, vol. 8, no. 1, pp. 76-80, 2001.

[7] S. Trichak, "Three-port vs standard four-port laparoscopic cholecystectomy," Surgical Endoscopy, vol. 17, no. 9, pp. 14341436, 2003.

[8] C. R. Wheeless, "A rapid, inexpensive and effective method of surgical sterilization by laparoscopy," The Journal of Reproductive Medicine, vol. 3, no. 5, pp. 65-69, 1969.

[9] M. A. Pelosi and M. A. Pelosi III, "Laparoscopic appendectomy using a single umbilical puncture (minilaparoscopy)," Journal of Reproductive Medicine for the Obstetrician and Gynecologist, vol. 37, no. 7, pp. 588-594, 1992.

[10] G. Navarra, E. Pozza, S. Occhionorelli, P. Carcoforo, and I. Donini, "One-wound laparoscopic cholecystectomy," British Journal of Surgery, vol. 84, no. 5, p. 695, 1997.

[11] G. Piskun and S. Rajpal, "Transumbilical laparoscopic cholecystectomy utilizes no incisions outside the umbilicus," Journal of Laparoendoscopic and Advanced Surgical Techniques A, vol. 9, no. 4, pp. 361-364, 1999.

[12] J. D. Raman, K. Bensalah, A. Bagrodia, J. M. Stern, and J. A. Cadeddu, "Laboratory and clinical development of single keyhole umbilical nephrectomy," Urology, vol. 70, no. 6, pp. 1039-1042, 2007. 
[13] H.-C. Tai, C.-D. Lin, C.-C. Wu, Y.-C. Tsai, and S. S.-D. Yang, "Homemade transumbilical port: an alternative access for laparoendoscopic single-site surgery (LESS)," Surgical Endoscopy, vol. 24, no. 3, pp. 705-708, 2010.

[14] D. Bhandarkar, G. Mittal, R. Shah, A. Katara, and T. E. Udwadia, "Single-incision laparoscopic cholecystectomy: how i do it?" Journal of Minimal Access Surgery, vol. 7, no. 1, pp. 17-23, 2011.

[15] J. Leroy, R. A. Cahill, M. Asakuma, B. Dallemagne, and J. Marescaux, "Single-access laparoscopic sigmoidectomy as definitive surgical management of prior diverticulitis in a human patient," Archives of Surgery, vol. 144, no. 2, pp. 173179, 2009.

[16] A. Rané, P. Rao, and P. Rao, "Single-port-access nephrectomy and other laparoscopic urologic procedures using a novel laparoscopic port (R-port)," Urology, vol. 72, no. 2, pp. 260 263, 2008.

[17] Z. Mehmood, A. Subhan, N. Ali et al., "Four port versus single incision laparoscopic cholecystectomy," Journal of Surgery Pakistan, vol. 15, no. 3, 2010.

[18] S. I. Choi, K. Y. Lee, S. J. Park, and S. H. Lee, "Single port laparoscopic right hemicolectomy with D3 dissection for advanced colon cancer," World Journal of Gastroenterology, vol. 16, no. 2, pp. 275-278, 2010.

[19] W. Jeong, H. G Jeon, H. S. Yu et al., "Embryonic-natural orifice transluminal endoscopic surgery nephrectomy," Korean Journal of Urology, vol. 50, no. 6, pp. 609-612, 2009.

[20] Y. W. Jung, S. W. Kim, and Y. T. Kim, "Recent advances of robotic surgery and single port laparoscopy in gynecologic oncology," Journal of Gynecologic Oncology, vol. 20, no. 3, pp. 137-144, 2009.

[21] M. S. Cho, B. S. Min, Y. K. Hong, and W. J. Lee, "Single-site versus conventional laparoscopic appendectomy: comparison of short-term operative outcomes," Surgical Endoscopy, vol. 25, no. 1, pp. 36-40, 2011.

[22] K. C. Wen, K. Y. Lin, Y. Chen, Y. F. Lin, K. S. Wen, and Y. H. Uen, "Feasibility of single-port laparoscopic cholecystectomy using a homemade laparoscopic port: a clinical report of 50 cases," Surgical Endoscopy, vol. 25, no. 3, pp. 879-882, 2011. 


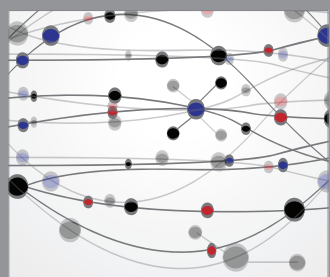

The Scientific World Journal
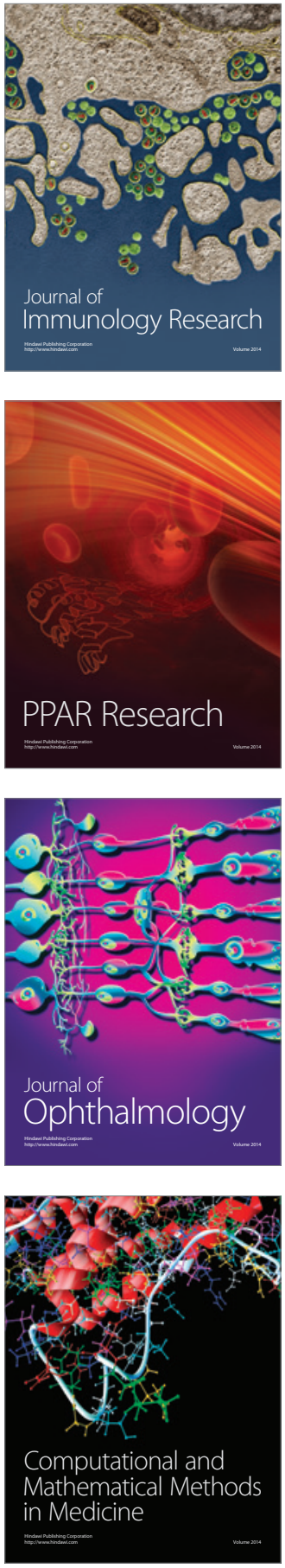

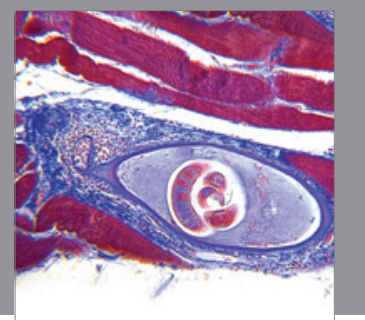

Gastroenterology

Research and Practice
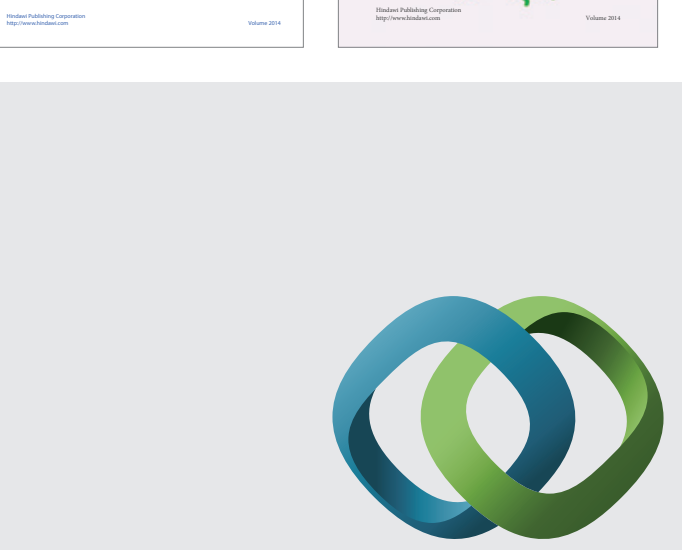

\section{Hindawi}

Submit your manuscripts at

http://www.hindawi.com
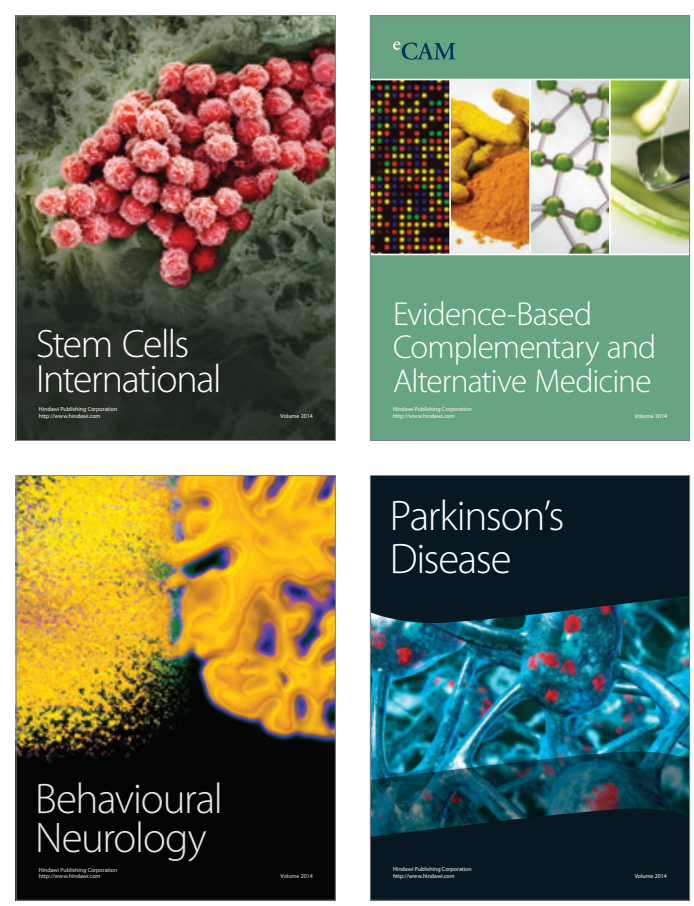

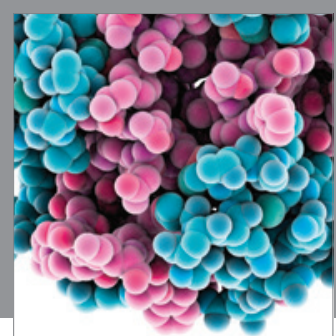

Journal of
Diabetes Research

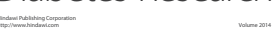

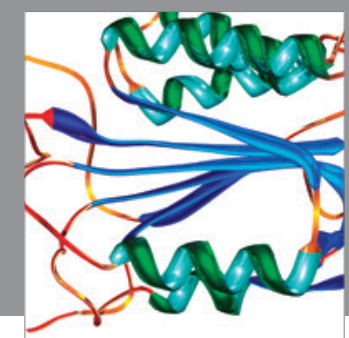

Disease Markers
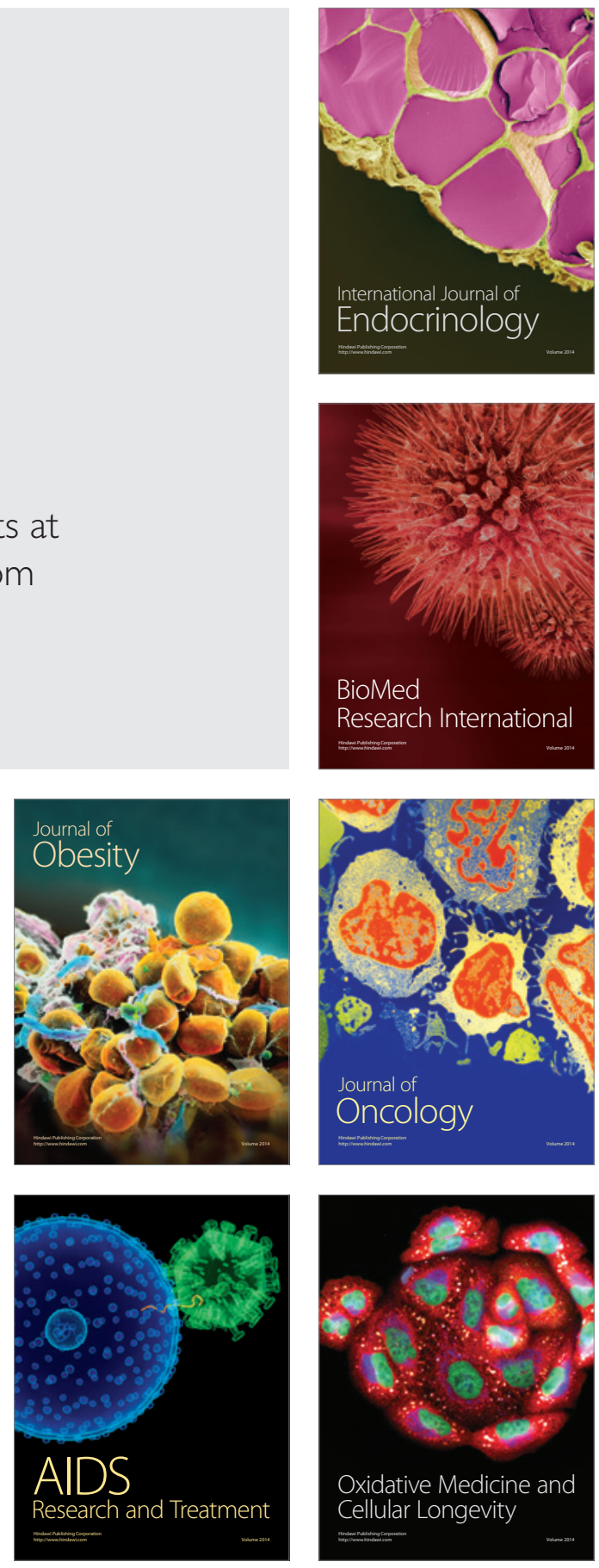